\title{
Óleo de citronela no controle do carrapato de bovinos
}

\author{
Citronella oil on the control of catle ticks
}

\begin{abstract}
Clair Jorge Olivo ${ }^{I}$ Nelcy Madruga de CarvalhoII José Henrique Souza da Silva ${ }^{\text {II }}$ Fernanda Flores Vogel ${ }^{\mathrm{II}}$ Péricles Massariol ${ }^{\mathrm{IV}}$ Gilmar Meinerz $^{\mathrm{V}}$ Carlos Agnolin $^{\mathrm{V}}$ Ademir Farias Morel $^{\mathrm{VI}}$ Luiz Volnei Viau ${ }^{\mathrm{VI}}$
\end{abstract}

\section{RESUMO}

O objetivo deste estudo foi avaliar o efeito in vivo $e$ in vitro do óleo de citronela (Cymbopogon nardus (L.) Rendle) no carrapato de bovinos (Boophilus microplus). O óleo foi obtido de folhas frescas pelo processo de destilação e o rendimento foi de $0,7 \%$. Para as experimentações in vitro foram usadas sete $(0 ; 0,5 ; 1,0 ; 10,0 ; 25,0 ; 50,0 ; 100,0 \%) e$ nove $(0,1 ; 0,25 ; 0,5 ; 1,0 ; 2,0 ; 10,0 ; 25,0 ; 50,0 ; 100 \%)$ concentrações de óleo de citronela, sendo testadas em fêmeas ingurgitadas. A eficácia observada foi de $0 ; 44,2 ; 92,1 ; 85,6$; 87,$8 ; 87,0 ; 88,9$ e de 0,$7 ; 2,8 ; 51,6 ; 79,3 ; 81,0 ; 87,1 ; 86,7$; $89,5 \%$, respectivamente. Os resultados sugerem que o óleo de citronela pode ser usado no controle do carrapato bovino.

Palavras-chave: Acaricida, amitraz, Boophilus microplus, Cymbopogon nardus, fitoterápico.

\section{ABSTRACT}

This research was aimed at evaluating in vitro and in vivo effects of citronella (Cymbopogon nardus (L.) Rendle) oil on catle ticks. The volatile oil was obtained from de fresh leaves of citronella grass by steam destilation and the yelds was $0.7 \%$. Seven $(0 ; 0.5 ; 1.0 ; 10.0 ; 25.0 ; 50.0 ; 100.0 \%)$ and nine $(0.1 ; 0.25 ; 0.5 ; 1.0 ; 2.0 ; 10.0 ; 25.0 ; 50.0 ; 100.0 \%)$ concentrations of citonella oil were used on in vitro trials with engorged female ticks. The efficacy control ticks was 0; 44.2; 92.1 ; 85.6; 87.8; 87.0; 88.9 and $0 ; 0.7 ; 2.8 ; 51.6$; 79.3; $81.0 ; 87.1 ; 86.789 .5 \%$, respectively. These sugest that the citronella oil can be practical used for controlling of catle ticks.
Key words: Acaricide, amitraz, Boophilus microplus, Cymbopogon nardus, phytotherapic.

\section{INTRODUÇÃO}

As perdas econômicas causadas por parasitas externos em rebanhos bovinos no Brasil superam a cifra de 2 bilhões de dólares ao ano. Desse montante, $75 \%$ é atribuídos ao carrapato. As demais parasitoses (mosca-dos-chifres, berne, miíases, moscados-estábulos) somadas correspondem aos 25\% restantes (GRISI et al., 2002).

O controle das parasitoses, basicamente, tem sido feito com produtos químicos que também acarretam malefícios aos organismos parasitados, ao homem, que consome os produtos de origem animal, e ao animal (CHAGAS et al., 2003). Agrega-se a esses problemas o desenvolvimento de resistência aos produtos químicos utilizados (FURLONG et al., 2004), especialmente em rebanhos de bovinos leiteiros (OLIVEIRA \& AZEVEDO, 2002). A indústria, por sua vez, tem hesitado em investir na pesquisa de novos defensivos químicos, considerando-se que o tempo de comercialização de um novo produto é de difícil cálculo, mas certamente limitado em função da rápida aquisição de resistência (CHAGAS et al., 2002).

'Departamento de Zootecnia (DZ), Universidade Federal de Santa Maria (UFSM), 97105-900, Santa Maria, RS, Brasil. Email: clairo@ccr.usfm.br. Autor para correspondência.

"DZ, UFSM, Santa Maria, RS, Brasil.

IIIDepartamento de Medicina Veterinária Preventiva, UFSM, Santa Maria, RS, Brasil.

${ }^{\mathrm{IV}}$ Curso de Pós-graduação em Zootecnia, UFSM, Santa Maria, RS, Brasil.

${ }^{\mathrm{v} C u r s o}$ de Zootecnia, UFSM, Santa Maria, RS, Brasil.

${ }^{\mathrm{V}}$ Departamento de Química, UFSM, Santa Maria, RS, Brasil.

VIIPólo Oleoquímico, Universidade Regional do Noroeste do Rio Grande do Sul (UNIJUí), Ijuí, RS, Brasil. 
Nesse contexto, a fitoterapia é considerada uma alternativa importante no controle de parasitas, podendo reduzir os impactos econômicos e ambientais ao uso de pesticidas sintéticos. Agrega-se também a isso que um aumento na produção de alimentos orgânicos no Brasil e no mundo implica terem-se alternativas mais eficientes no controle de parasitas, já que este tipo de agricultura não permite o uso de pesticidas. Ressalta-se também que o uso de fitoterápicos em sistemas convencionais de produção, como parte da estratégia de controle de parasitas, pode elevar a vida útil dos produtos químicos (VIEIRA et al., 1999).

Embora havia uma grande variabilidade de espécies de plantas, pesquisas sobre seu uso no controle de parasitas de animais, especialmente, são escassas, havendo carência de informações complementares em relação às condições de produção, à época de colheita e às quantidades e partes da planta utilizadas na elaboração dos produtos (HEIMERDINGER et al., 2006).

Dentre as plantas indicadas para o controle de insetos, destaca-se a citronela (Cymbopogon nardus L. Rendle), cultivada em regiões tropicais e subtropicais. O óleo extraído de suas folhas, frescas ou parcialmente dessecadas, é usado como repelente de mosquitos. Essa propriedade é atribuída à presença de substâncias voláteis em suas folhas, como citronelal, eugenol, geramiol e limoneno, entre outras, denominadas de um modo geral como monoterpenos (SHASANY et al., 2000). Pesquisas conduzidas com o óleo dessa planta demonstraram sua ação como inseticida e de repelência contra mosquitos e moscas (RAJA et al., 2001). Estudos feitos com destilados de folhas de citronela demonstraram elevada ação carrapaticida, tanto em larvas quanto em fêmeas adultas, sendo usados, no entanto, óleo puro e níveis considerados altos de óleo em etanol, de 1:4 (CHUNGSAMARNYART \& JIWAJINDA, 1992).

Assim, o objetivo deste trabalho foi avaliar a eficácia do óleo de citronela como ectoparasiticida em bovinos leiteiros a partir de experimentações in vitro, envolvendo equipe multidisciplinar.

\section{MATERIAL E MÉTODOS}

Para a pesquisa, foram coletadas fêmeas ingurgitadas com comprimento superior a $4 \mathrm{~mm}$, colhidas de animais da raça Holandesa, naturalmente infestados, pertencentes ao Laboratório de Bovinocultura de Leite (DZ, UFSM).

Os óleos de citronela testados são oriundos da mesorregião Noroeste do Rio Grande do Sul. O óleo obtido da parte aérea de plantas frescas apresentou um rendimento de $0,7 \%$, aproximadamente. A análise cromatográfica dos produtos foi realizada em laboratório da Central Analítica do Pólo Oleoquímico (UNIJUÍ), apresentando, em média, como princípios ativos mais importantes, 50,07; 7,93; 13,87; 28,13\% para o citronelal, citronelol, geraniol e outros compostos, respectivamente. Os tratamentos (Experimento I) foram constituídos por diferentes concentrações do óleo de citronela (0; 0,5; 1,0; 10,0; 25,0; 50,0 e 100,0\%), oriundo de um produtor que industrializa o produto e está ligado à rede de produtores de óleos essenciais do Pólo Oleoquímico (Três Passos, RS). Para o grupo controle (zero) e para a complementação das demais soluções usou-se água destilada.

As teleóginas foram colocadas aleatoriamente em grupos de 10 em cada placa de Petry, sendo usadas três placas/tratamento, submetidas ao teste do biocarrapaticidograma (à semelhança de DRUMMOND et al., 1973), em três imersões, com intervalos de 24 horas, no Laboratório de Parasitologia da UFSM.

O delineamento experimental utilizado foi o inteiramente casualizado, com três repetições (placas de Petry). Os resultados, referentes à inibição da postura, eclosão das larvas e a eficiência dos produtos em carrapatos foram submetidos à análise de variância e à função logarítmica de base 10. Os dados foram submetidos à análise de regressão polinomial em função das diferentes soluções, constituídas pelos níveis de citronela. Foi utilizado o programa estatístico SAS (1997).

Para o experimento II, foram constituídos tratamentos com diferentes concentrações de citronela (0; 0,$1 ; 0,25 ; 0,5 ; 1,0 ; 10,0 ; 25,0 ; 50,0$ e 100,0\%), usandose material de duas fontes (uma oriunda do produtor rural, utilizada no experimento I, e outra do Laboratório de Óleos Essenciais (UNIJUÍ).

Para a avaliação dos óleos seguiu-se a mesma metodologia adotada no experimento I. O delineamento experimental utilizado foi o de blocos casualizados com três repetições (placas de Petry)/ bloco (seis placas/tratamento). O critério de bloqueamento foram as fontes de produção de óleo. Para a análise dos dados usou-se metodologia similar à adotada no experimento I (SAS, 1997).

\section{RESULTADOS E DISCUSSÃO}

Os resultados referentes à eficácia dos produtos pelo teste do biocarrapaticidograma encontram-se na tabela 1 . No experimento I observa-se que, com relação à inibição de postura, não houve diferença significativa $(\mathrm{P}>0,05)$ entre as soluções

Ciência Rural, v.38, n.2, mar-abr, 2008. 
Tabela 1 - Médias porcentuais de inibição das posturas, da eclosão de larvas e da eficiência das soluções constituídas pelo controle e por diferentes concentrações de óleo de citronela (Cymbopogon nardus L. Rendle) em teleóginas de Boophilus microplus pela técnica do biocarrapatidograma. Santa Maria, RS, 2006.

\begin{tabular}{lccc}
\hline \multicolumn{4}{c}{ Experimento 1} \\
Tratamentos (\%) & $\begin{array}{c}\text { Inibição da } \\
\text { postura (\%) }\end{array}$ & $\begin{array}{c}\text { Eclosão da } \\
\text { postura (\%) }\end{array}$ & $\begin{array}{c}\text { Eficácia do } \\
\text { produto (\%) }\end{array}$ \\
\hline 0 (controle) & $3,33^{\mathrm{a}}$ & 98,3 & - \\
0,5 & $60,0^{\mathrm{ab}}$ & 98,3 & 44,2 \\
1,0 & $90,0^{\mathrm{a}}$ & 78,3 & 92,1 \\
10,0 & $90,0^{\mathrm{a}}$ & 30,0 & 85,6 \\
25,0 & $90,0^{\mathrm{a}}$ & 65,0 & 87,8 \\
50,0 & $86,7^{\mathrm{a}}$ & 65,0 & 87,0 \\
100 & $90,0^{\mathrm{a}}$ & 53,3 & 88,9 \\
& Experimento 2 & \\
0 (controle) & $16,6^{\mathrm{c}}$ & 99,1 & - \\
0,1 & $5,0^{\mathrm{c}}$ & 100,0 & $0,71^{\mathrm{c}}$ \\
0,25 & $5,0^{\mathrm{c}}$ & 98,3 & $2,85^{\mathrm{c}}$ \\
0,5 & $55,0^{\mathrm{b}}$ & 97,5 & $51,6^{\mathrm{c}}$ \\
1,0 & $65,0^{\mathrm{ab}}$ & 87,5 & $79,3^{\mathrm{b}}$ \\
10,0 & $73,3^{\mathrm{ab}}$ & 58,3 & $81,0^{\mathrm{a}}$ \\
25,0 & $85,0^{\mathrm{a}}$ & 76,0 & $87,1^{\mathrm{a}}$ \\
50,0 & $78,3^{\mathrm{ab}}$ & 68,3 & $86,7^{\mathrm{a}}$ \\
100,0 & $88,3^{\mathrm{a}}$ & 58,3 & $89,5^{\mathrm{a}}$ \\
\hline & & &
\end{tabular}

$\mathrm{P}<0,01$, nas colunas, em cada experimento.

Coeficientes de Variação: 20,01 e 16,51; 21,20 e 30,93; 26,42 e 28,58\% para inibição da postura, eclosão de larvas e eficácia do produto, para os experimentos 1 e 2 , respectivamente.

constituídas pelas diferentes concentrações de óleo de citronela e o nível de $0,5 \%$ não diferiu do grupo controle. Para a porcentagem de eclosão das larvas e eficácia dos tratamentos não foram encontradas diferenças $(\mathrm{P}<0,05)$ entre as soluções. Os dados, transformados por função logarítmica e submetidos à análise de regressão (Figura 1), demonstraram efeito cúbico $(\mathrm{P}<0,10)$ para inibição da postura no decorrer dos dias ( $\mathrm{x}$ ) de avaliação $\left(\mathrm{Y}=5,98+0,31 \mathrm{x}-0,0077 \mathrm{x}^{2}+\right.$ $\left.0,00005 x^{3}\right)$ e para eficácia $\left(Y=67,55+1,89 x-0,044 x^{2}+\right.$ $\left.0,00027 x^{3}\right)$.

No experimento II, no qual foram usadas duas fontes de óleo, verificou-se que, considerandose o valor médio dos resultados em relação às concentrações utilizadas, houve diferença $(\mathrm{P}<0,05)$ quanto à inibição de postura entre o produto oriundo do Pólo Oleoquímico (44,7\%) e do produtor rural $(60,7 \%)$. Para a eclosão das larvas e a eficiência de controle não houve diferença entre elas $(\mathrm{P}>0,05)$, sendo de 89,62 e 51,06\% e de 76,11 e 54,04\%, respectivamente.
Considerando-se os tratamentos utilizados, observa-se que as concentrações de 100, 50, 25, 10 e $1 \%$ proporcionaram maior inibição de postura de ovos pelas fêmeas ingurgitadas, comprovando-se um efeito ascendente $\left(\mathrm{Y}=23,13+1,99 \mathrm{x}-0,013 \mathrm{x}^{2} ; \mathrm{P}<0,05\right)$, a medida que foram aumentadas as concentrações (Figura 1). Para a eclosão das larvas houve similaridade $(\mathrm{P}>0,05)$ entre os tratamentos testados, havendo um comportamento análogo ao resultado do Experimento I. Já para a eficiência de controle, as concentrações de 0,$1 ; 0,25$ e $0,5 \%$ foram inferiores $(\mathrm{P}<0,05)$ à de $1 \%$ e esta inferior aos demais níveis, verificando-se um comportamento cúbico ascendente $(\mathrm{Y}=24,72+6,1 \mathrm{x}$ $\left.0,143 x^{2}+0,0008 x^{3}\right)$. Observa-se que, tanto no Experimento I quanto no II, houve um aumento no controle do carrapato a medida em que as concentrações de óleo foram elevadas. Pondera-se, no entanto, sobre os modelos de regressão encontrados (não lineares), e que as concentrações de óleo de citronela $(0,5$ e $1 \%)$ apresentam resultados proporcionalmente maiores que os níveis mais concentrados do produto. Possivelmente, esse resultado deve-se ao fenômeno chamado de apassivação, onde o produto (concentrado) é absorvido, mas depois forma um filme apassivador, barrando a passagem do óleo. Quando ele está diluído, este filme não se forma e a penetração ocorre mais lentamente, mas de maneira mais devastadora (CHAGAS et al., 2003). Para que o produto bioativo seja absorvido, ele precisa ser hidrofílico e lipolítico, pois os artrópodes possuem esses meios de absorção (ODHIAMBO, 1982). O óleo puro é somente lipolítico, possuindo assim menor absorção.

A ação acaricida deste produto também foi comprovada por CHUNGSAMARNYART \& JIWAJINDA (1992) que, utilizando o óleo de citronela a 12,5; 8,3 e 7,1\%, diluído em etanol, verificaram ação larvicida de 95,7; 92,7 e 58,1\%, respectivamente. Avaliações feitas com óleo de citronela de Java (Cymbopogon winterianus) demonstraram uma eficiência de $50 \%$ no controle de teleóginas e larvas, usando concentrações de 6,1 e 4,1\%, respectivamente. Foi verificado, ainda, que não houve postura quando as teleóginas foram tratadas com esse óleo na concentração de $10 \%$ e tão pouco houve eclosão das larvas destas teleóginas na concentração de 7,14\% (MARTINS, 2006).

Esta ação deve-se ao princípio ativo citronelal, de comprovada ação inseticida e acaricida (CHAGAS et al., 2002). Pesquisa conduzida com citronelal e geraniol, estudados separadamente, demonstrou uma ação acaricida significativamente maior que o citronelol, indicando haver uma ação compartilhada entre eles. (MARTINS, 2006) 


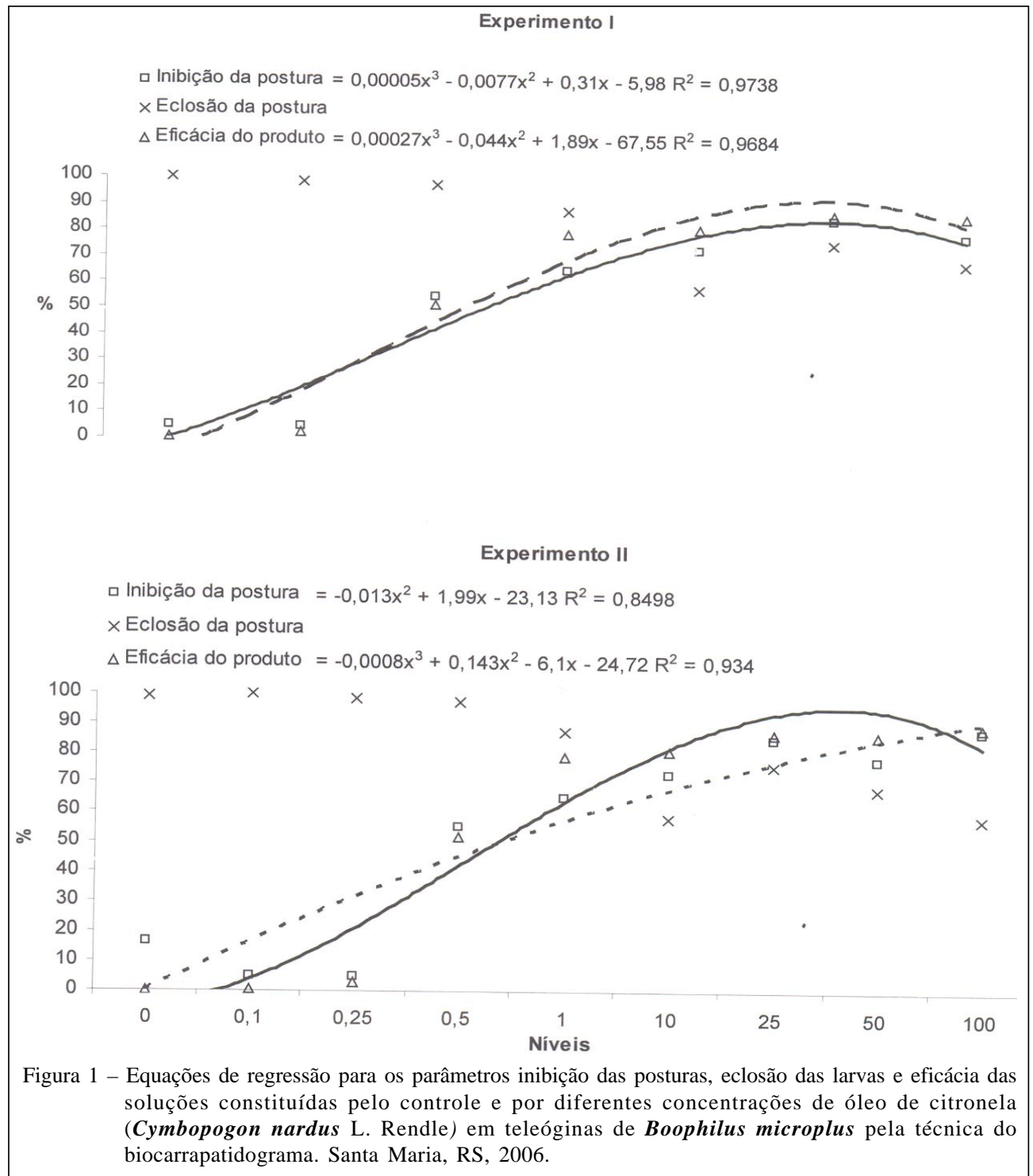

Considerando-se os resultados obtidos e que as soluções feitas com extratos de plantas tendem a apresentar menor toxicidade aos mamíferos, rápida degradação e desenvolvimento lento de resistência (CHUNGSAMARNYART \& JIWAJINDA, 1992), os resultados podem ser importantes como estratégia de controle do carrapato em diferentes sistemas de produção. Constata-se, no entanto, a necessidade de se realizar novos estudos para otimizar o óleo de citronela, especialmente na avaliação com emulsionantes, na forma e no período de utilização do produto constituído.

\section{CONCLUSÕES}

As experimentações demonstram que o óleo de citronela apresenta atividade acaricida, havendo comportamento similar entre as soluções elaboradas com produtos de diferentes fontes de produção.

\section{REFERÊNCIAS}

CHAGAS, A.C.S. et al. Sensibilidade do carrapato Boophilus microplus a solventes. Ciência Rural, v.33, n.1, p.109-114, 2003.

CHAGAS, A.C.S. et al. Efeito acaricida de óleos essenciais e concentrados emulsionáveis de Eucalyptus spp em Boophilus microplus. Brazilian Journal of Veterinary Research and Animal Science, v.39, n.5, p.247-253, 2002.

CHUNGSAMARNYART, N.; JIWAJINDA, S. Acaricidal activity of volatile oil from lemon and citronella grasses on tropical cattle ticks. Kasetsart Journal, v.26, p.46-51, 1992. 
FURLONG, J. Controle estratégico do carrapato dos bovinos. A Hora Veterinária, RS, v.23, n.137, p.53-56, 2004.

GRISI, L. et al. Impacto econômico das principais ectoparasitoses em bovinos no Brasil. A Hora Veterinária, v.21, n.125, p.8-10, 2002.

HEIMERDINGER, A. et al. Extrato alcoólico de capim-cidreira no controle do Boophilus microplus em bovinos. Revista Brasileira de Parasitologia Veterinária, v.15, n.1, p.3739, 2006.

MARTINS, R.M. Estudo in vitro da ação acaricida do óleo essencial da gramínea Citronela de Java (Cymbopogon winterianus Jowitt) no carrapato Boophilus microplus. Revista Brasileira de Plantas Medicinais, v.8, n.2, p.71-78, 2006.

ODHIAMBO, T.R. Current themes in tropical science: physiology of ticks. Oxford: Pergamon, 1982. v.1, p.508.
OLIVEIRA, A.A; AZEVEDO, H.C. Resistência do carrapato Boophilus microplus a carrapaticidas em bovinos de leite na região dos tabuleiros costeiros de Sergipe. Revista Científica Rural, v.7, n.2, p.64-71, 2002.

SAS, Statistical Analisys System: User's Guide, Stat. 2.ed. Cary, 1997. 456p.

SHASANY, A.K. et al. Phenotypic and RAPD diversity among Cymbopogon Winterianus Jowitt accessions in relation to Cymbopogon nardus Rendle. Genetic Resources and Crop Evolution, v.47, n.5, p.553-559, 2000.

RAJA, N. et al. Effect of volatile oils in protecting stored Vigna unguiculata (L.) Walpers against Callosobruchus maculatus (F.) (Coleóptera: Bruchidae) infestation. Journal of Stored Products Research, v.37, n.2, p.127-132, 2000.

VIEIRA, L.S; CAVALCANTE, A.C.R. Resistência antihelmíntica em rebanhos caprinos no Estado do Ceará. Pesquisa Veterinária Brasileira, v.19, n.3, p.99-103, 1999. 